\title{
Identification of deliberate catheter motion at the left atrial posterior wall during pulmonary vein isolation: Validity of respiratory motion adjustment
}

\author{
David Tomlinson ${ }^{1}$, Katie Biscombe ${ }^{2}$, John True ${ }^{2}$, Joanne Hosking ${ }^{2}$, and Adam Streeter ${ }^{2}$ \\ ${ }^{1}$ Plymouth Hospitals NHS Trust \\ ${ }^{2}$ Plymouth University Peninsula Schools of Medicine and Dentistry
}

November 26, 2020

\begin{abstract}
Background During automated radiofrequency (RF) annotation-guided pulmonary vein isolation (PVI), respiratory motion adjustment (RMA) is recommended, yet lacks in vivo validation. Methods Following contact force (CF) PVI (continuous $\mathrm{RF}, 30 \mathrm{~W}$ ) using general anaesthesia and automated RF annotation-guidance (VISITAG: force-over-time 100\% minimum 1g; 2mm position stability; ACCURESP RMA "off") in 25 patients, we retrospectively examined RMA settings "on" versus "off" at the left atrial posterior wall (LAPW). Results Respiratory motion detection occurred in 8, permitting offline retrospective comparison of RMA settings. Significant differences in LAPW RF auto-annotation occurred according to RMA setting, with curves displaying catheter position, $\mathrm{CF}$ and impedance data indicating "best-fit" for catheter motion detection using RMA "off". Comparing RMA "on" versus "off", respectively: Total annotated sites 82 versus 98; median RF duration per-site 13.3s versus 10.6s $(\mathrm{p}<0.0001)$; median force time integral $177 \mathrm{~g} . \mathrm{s}$ versus $130 \mathrm{~g} . \mathrm{s}$ ( $\mathrm{p}=0.0002)$; mean inter-tag distance (ITD) 6.0mm versus $4.8 \mathrm{~mm}(\mathrm{p}=0.002)$. Considering LAPW annotated site 1-to-2 transitions resulting from deliberate catheter movement, 3 concurrent with inadvertent $\mathrm{Og}$ CF demonstrated $<0.6$ s difference in RF duration. However, 13 deliberate catheter movements during constant tissue contact (ITD range $2.1-7.0 \mathrm{~mm}$ ) demonstrated (mean) site-1 RF duration difference 3.7s (range: 1.3 to $11.3 \mathrm{~s}$ ): considering multiple measures of catheter position instability, the appropriate indication of deliberate catheter motion occurred with RMA "off" in all. Conclusions ACCURESP respiratory motion adjustment importantly delayed the identification of deliberate and clinically relevant catheter motion during LAPW RF delivery, rendering auto-annotated RF display invalid. Operators seeking greater accuracy during auto-annotated RF delivery should avoid RMA use.
\end{abstract}

Title Identification of deliberate catheter motion at the left atrial posterior wall during pulmonary vein isolation: Validity of respiratory motion adjustment

Authors: David R. Tomlinson BM BSc MD ${ }^{1}$, Katie Biscombe ${ }^{2}$, John True BSc MSc ${ }^{2}$, Joanne Hosking BSc $\mathrm{PhD}^{2}$ and Adam J. Streeter MSc $\mathrm{PhD}^{2}$

Corresponding address: ${ }^{1}$ University Hospitals Plymouth NHS Trust, South West Cardiothoracic Centre, Derriford Hospital, Plymouth, UK, PL6 8DH

${ }^{2}$ Department of Medical Statistics, Plymouth University Peninsula Schools of Medicine and Dentistry, ITTC Building 1, Plymouth Science Park, Plymouth, Devon PL6 8BX, UK.

All authors take responsibility for all aspects of the reliability and freedom from bias of the data presented and their discussed interpretation.

Email: david.tomlinson1@nhs.net 
Sources of Funding: I am grateful to the "Sarkar Research and Training" charitable fund, University Hospitals Plymouth NHS Trust for a donation of $£ 1000$, funding the R software code development and extended data analyses by teams at the Department of Medical Statistics, Plymouth University Peninsula Schools of Medicine and Dentistry.

Word count: Abstract 250, body text 3563

Conflict of interest: None declared

Keywords: atrial fibrillation; contact force catheter ablation; pulmonary vein isolation; respiratory motion adjustment; automated radiofrequency annotation

\section{Abstract \\ Background}

During automated radiofrequency (RF) annotation-guided pulmonary vein isolation (PVI), respiratory motion adjustment (RMA) is recommended, yet lacks in vivo validation.

\section{Methods}

Following contact force (CF) PVI (continuous RF, 30W) using general anaesthesia and automated RF annotation-guidance (VISITAG: force-over-time 100\% minimum 1g; 2mm position stability; ACCURESP RMA "off") in 25 patients, we retrospectively examined RMA settings "on" versus "off" at the left atrial posterior wall (LAPW).

\section{Results}

Respiratory motion detection occurred in 8, permitting offline retrospective comparison of RMA settings. Significant differences in LAPW RF auto-annotation occurred according to RMA setting, with curves displaying catheter position, CF and impedance data indicating "best-fit" for catheter motion detection using RMA "off". Comparing RMA "on" versus "off", respectively: Total annotated sites 82 versus 98; median RF duration per-site $13.3 \mathrm{~s}$ versus $10.6 \mathrm{~s}(\mathrm{p}<0.0001)$; median force time integral $177 \mathrm{~g}$.s versus $130 \mathrm{~g} . \mathrm{s}(\mathrm{p}=0.0002)$; mean inter-tag distance (ITD) $6.0 \mathrm{~mm}$ versus $4.8 \mathrm{~mm}(\mathrm{p}=0.002)$. Considering LAPW annotated site 1-to-2 transitions resulting from deliberate catheter movement, 3 concurrent with inadvertent $0 \mathrm{~g}$ CF demonstrated $<0.6 \mathrm{~s}$ difference in RF duration. However, 13 deliberate catheter movements during constant tissue contact (ITD range $2.1-7.0 \mathrm{~mm}$ ) demonstrated (mean) site-1 RF duration difference $3.7 \mathrm{~s}$ (range: -1.3 to $11.3 \mathrm{~s}$ ): considering multiple measures of catheter position instability, the appropriate indication of deliberate catheter motion occurred with RMA "off" in all.

\section{Conclusions}

ACCURESP respiratory motion adjustment importantly delayed the identification of deliberate and clinically relevant catheter motion during LAPW RF delivery, rendering auto-annotated RF display invalid. Operators seeking greater accuracy during auto-annotated RF delivery should avoid RMA use.

\section{Introduction}

The central importance of electrical pulmonary vein isolation (PVI) towards eliminating atrial fibrillation (AF) is well-established. ${ }^{1,2}$ Important progress towards improving procedural success was recently reported using site-specific contact force (CF) radiofrequency (RF) energy delivery targets, defined using a weighted formula incorporating RF power, CF and duration (Ablation Index, AI, Biosense Webster Inc., Diamond Bar, CA). ${ }^{3,4}$ This entire experimental approach was underpinned by a process of automated RF annotation (VISITAG Module, Biosense Webster), targeting a minimum inter-tag distance (ITD) of 6mm. However, this may represent an imperfect description of a genuinely reproducible PVI protocol, since - employing either conscious sedation or general anaesthesia (GA) with intermittent positive pressure ventilation (IPPV) - these studies ${ }^{5}$ routinely applied end-expiratory respiratory motion adjustment (RMA) to automated RF annotation settings (i.e. ACCURESP "on"). Importantly, such RMA use remains without in vivo validation. 
Therefore, in view of the importance of accurate RF auto-annotation at the LAPW due to the site-specific risk of atrio-oesophageal fistula (AEF), we retrospectively investigated the effects of RMA use on LAPW automated RF annotation, following CF-guided PVI.

\section{Methods}

Single operator CF and VISITAG Module-guided PVI was performed using a previously reported standardised protocol ${ }^{6}$ in consecutive, unselected adult patients with symptomatic AF undergoing first-time PVI according to current treatment indications. ${ }^{7}$ Briefly, all procedures were undertaken using GA and IPPV, with respiratory motion "training" undertaken pre-ablation and applied as required to complete the CARTO $^{(\mathrm{r})} 3$ geometry (V.3, Biosense Webster). Specifically, respiratory motion training was first performed with a LASSO ${ }^{(\mathrm{r})} \mathrm{Nav}$ catheter (Biosense Webster, 2-5-2mm inter-electrode spacing) within the right superior $\mathrm{PV}$. If there was insufficient respiratory motion to trigger its detection, the catheter was placed within the left inferior PV and respiratory motion training re-checked. The tidal volume was never deliberately increased, so respiratory motion detection was negative in some cases. When respiratory motion detection was triggered, RMA (ACCURESP) remained "off"; i.e. RMA was never applied to automated RF annotation during PVI.

Temperature-controlled RF at $30 \mathrm{~W}\left(17 \mathrm{ml} / \mathrm{min}\right.$ irrigation, $\left.48^{\circ} \mathrm{C}\right)$ was delivered via a SmartTouch(r) (Biosense Webster) catheter using Agilis NxT sheath (Abbott, St Paul, MN) support during proximal pole CS pacing at $600 \mathrm{~ms}$. Filter preferences for automated RF annotation were: Positional stability range $2 \mathrm{~mm}$, tag display duration $3 \mathrm{~s}$; force-over-time $100 \%$ minimum $1 \mathrm{~g}$ (the latter derived from a previous study ${ }^{8}$ and designed to ensure on-going RF annotation only in the presence of constant catheter-tissue contact). RF delivery was guided by automated RF annotation, with the preferred site of first RF application at the LAPW opposite each superior $\mathrm{PV} \sim 1 \mathrm{~cm}$ from the PV ostium. The target annotated RF duration at each first-ablated LAPW site, as well as any subsequent "RF ON" sites and at the carina (if ablated) was 15s, whereas $9-11 \mathrm{~s}$ was the target for all other sites consecutively annotated during continuous RF delivery. Following the required period of first-site annotated RF delivery, target ITD [?]6 $\mathrm{mm}$ was achieved predominantly during continuous RF application using deliberate, rapid movement of the catheter tip initiated via the Agilis sheath, aided by the distance measurement tool; point-by-point RF was also applied as necessary. Following completion of circumferential PVI (entrance and exit block), spontaneous recovery of PV conduction was assessed and eliminated during a minimum 20-minute wait; dormant recovery was evaluated and eliminated a minimum of 20 minutes after the last RF. Neither oesophageal luminal temperature monitoring nor post-ablation endoscopic evaluation was employed.

All cases where respiratory motion detection occurred were used to retrospectively assess the effects of RMA on automated RF annotation at the LAPW. VISITAG annotated RF and UE morphology change data were retrospectively collected; ablation naive LAPW sites were examined (i.e. first encirclement during continuous RF delivery, not including touch-up lesions), with annotated RF duration, mean CF, force time integral (FTI) and impedance drop data for each site obtained via the VISITAG export function. To examine the effects of RMA, RF data export was performed separately for ACCURESP "on" and "off": at each setting, ITD was determined on-line using the proprietary measurement tool. Retrospective UE analyses were performed using recorded signals (CARTOREPLAY, Biosense Webster) as previously described ${ }^{6}$ : electrograms were automatically deleted at 12-18 hours after case completion $\left(\right.$ a CARTO $^{(\mathrm{r})} 3$ system function), so UE morphology data was only obtained for the ACCURESP "off" setting. This work received IRB approval for publication as a retrospective service evaluation. All patients provided written, informed consent.

\section{Analysis}

The question was whether ACCURESP RMA "on" versus "off" settings resulted in significant differences in the number of annotated sites, individual site RF duration, ILD, total impedance drop, mean CF and FTI, at the LAPW. RMA "on" versus "off" annotation accuracy with respect to the time of deliberate catheter motion was assessed at all automated site 1-to-2 ablation catheter transitions (i.e. site 1-to-2 transitions at each of the left and right PVI encirclements) by visual inspection of reconstituted catheter position, CF and 
impedance curves. Ablation catheter motion associated with transition between annotated sites 1-to-2 was identified according to any of the following criteria:

(1) Site 1 annotation "end" due to a $0 \mathrm{~g}$ CF event (i.e. CF breaching the VISITAG Module force-over-time filter of $100 \%$ minimum $1 \mathrm{~g}$ );

(2) Inter-ablation site transition during constant catheter-tissue contact, but accompanied by UE morphology change from pure R at site- 1 completion, to RS at site- 2 onset (i.e. indicating movement from a site of TM ablation effect to an adjacent, ablation naive site), or

(3) Inter-ablation site transition during constant catheter-tissue contact, with pure R UE morphology at both site- 1 completion and site-2 onset (i.e. within the thermal penumbra of partial ablation effect ${ }^{9}$ ).

Subsequent LAPW annotated RF site-to-site transitions were similarly identified and described. VISITAG Module data comprising catheter tip x,y,z coordinates at $60 \mathrm{~Hz}$ and without RMA (i.e. from the "RawPositions" data file) were exported to $\mathrm{R}^{10}$ for analysis. The standard deviation (SD) over a rolling one second period - as utilised by $\mathrm{CARTO}^{(\mathrm{r})} 3$ system for position stability annotation - was calculated from the Euclidean distances shifted between each position. These "position stability" data were plotted according to the patient interface unit (PIU) system time, alongside CF at $20 \mathrm{~Hz}$ and impedance at $10 \mathrm{~Hz}$ (derived from the exported "ContactForceData" "AblationData" files, respectively) and end-expiration timing (from the "EndExperium" file). Finally, PIU start and stop times for annotated sites were obtained for each ACCURESP setting (from "AblationSites" files).

\section{Statistics}

Exported text files were converted to Excel and imported to GraphPad Prism version 4.03 (GraphPad Software, San Diego, CA). Comparisons were made between ACCURESP "on" and "off" settings based on the means and standard deviations of biophysical data (i.e. ILD, RF duration, CF, impedance drop and FTI), or medians along with the $1^{\text {st }}$ and $3^{\text {rd }}$ quartile (IQR), where these were determined to be skewed. Any differences between these RMA settings were tested using the unpaired t-test, or the Mann-Whitney test where a normal distribution could not be assumed. Pearson correlation coefficients were calculated to determine the strength of association between ACCURESP "off" ITD and the difference (ACCURESP "on" minus "off") in RF duration, FTI and ILD. In this exploratory analysis, significance was set at the $5 \%$ level.

\section{Results}

Twenty-five patients underwent first-time PVI as described, between November 2016 and May 2017: 13 persistent AF, $12 \mathrm{PAF} ; 19$ male (76\%); mean age 57 [SD: 14] years and mean $\mathrm{CHA}_{2} \mathrm{DS}_{2}$-VASc score 1.3 [SD: 1.3]. Complete PVI was achieved in all without spontaneous / dormant recovery of PV conduction, following mean 16.2 [SD: 3.1] minutes of RF, without procedural complications. The respiratory motiontriggering cohort comprised 8 cases (32\%); considering age, body mass index and RF duration required for case completion, there were no significant differences between cohorts with and without respiratory motion threshold triggering.

Comparing ACCURESP RMA "on" versus "off", the number of auto-annotated LAPW sites and total LAPW RF duration were 82 and 98, and 1091s and 1006s, respectively. Annotated RF data according to RMA setting are shown in table 1. For each group (i.e. left or right-sided), per-site RF duration and FTI were significantly greater with RMA "on" versus "off": i.e. left-sided mean RF duration 13.1s versus 9.9s ( $\mathrm{p}=0.0003)$ and median FTI 156g.s versus 114g.s ( $\mathrm{p}=0.0003$ ), respectively; right-sided mean RF duration $13.5 \mathrm{~s}$ versus $10.6 \mathrm{~s}(\mathrm{p}=0.006)$ and median FTI $228 \mathrm{~g} . \mathrm{s}$ versus $166 \mathrm{~g} . \mathrm{s}(\mathrm{p}=0.04)$, respectively. Analysis combining left and right sides also demonstrated significantly greater mean ITD with RMA "on"; i.e. 6.0mm versus $4.8 \mathrm{~mm}(\mathrm{p}=0.002)$.

Table 2 shows comparisons of annotated RF data at site- 1 according to RMA setting: At the left-side and comparing ACCURESP RMA "on" versus "off" the site 1-to-2 ITD (6.6mm versus 5.3mm, p=0.07), $\mathrm{RF}$ duration (16.0s versus 15.1s, $\mathrm{p}=0.16$ ) and FTI (185g.s versus $163 \mathrm{~g} . \mathrm{s}, \mathrm{p}=0.33$ ) were greater with RMA 
"on", but without statistical significance. At the right side the site 1-to-2 ITD was greater with RMA "on" ( $7.2 \mathrm{~mm}$ versus $5.0 \mathrm{~mm}, \mathrm{p}=0.13)$, but without statistical significance. Combined data analysis demonstrated that auto-annotated site 1-to-2 ITD was significantly greater with RMA "on" versus "off" (i.e. $6.7 \mathrm{~mm}$ versus $5.2 \mathrm{~mm}, \mathrm{p}=0.02)$, while the difference in site- $1 \mathrm{RF}$ duration $(15.7 \mathrm{~s}$ versus $15.1 \mathrm{~s}, \mathrm{p}=0.09)$ and FTI $(240 \mathrm{~g} . \mathrm{s}$ versus $198 \mathrm{~g} . \mathrm{s}, \mathrm{p}=0.38$ ) was not statistically significant.

\section{Analyses at sites of deliberate catheter motion: Auto-annotated site 1-to-2 transitions}

Comparing auto-annotated data (RMA "on" minus "off") at 3 site 1-to-2 transitions concurrent with loss of tissue contact (i.e. $0 \mathrm{~g} \mathrm{CF}$ ), the maximum difference in RF duration, FTI and ITD was $-0.6 \mathrm{~s}$, $-17 \mathrm{~g}$.s and $2.2 \mathrm{~mm}$ respectively, with no difference in impedance drop (table 3 and figure 1, plus supplementary figures $1-2)$.

Four site 1-to-2 transitions were effected with constant catheter-tissue contact and associated with UE morphology change from pure R (site-1 completion) to RS (site-2 onset). In this group, the maximum difference (RMA "on" minus "off") in RF duration, FTI, ITD and impedance drop was 11.3s, 139g.s, 2.6mm and 3.3 respectively (table 3), with the first indication of catheter movement represented via RMA "off" annotation in all cases (figure 2 and supplementary figures 3-5). The greatest difference occurred when site 1-to-2 ITD with RMA "off" was $4.1 \mathrm{~mm}$ (figure 2): at 15.2s following RF onset there was an abrupt increase in catheter position shift and SD, with a corresponding change in $\mathrm{CF}$ waveform indicating deliberate catheter motion "per protocol" at 15s, however while annotated site 1-to-2 transition according to RMA "off" coincided with these changes (blue vertical line), the RMA "on" timing of annotated site 1-to-2 transition occurred 11.3s later (red vertical line).

The remaining 9 deliberate site 1-to-2 transitions during constant catheter-tissue contact were associated with continuous pure R UE morphology at both site- 1 end and site- 2 onset. Comparison of annotated data (RMA "on" minus "off") demonstrated a difference in site-1 RF duration $>1$ s in 8 ; range $-1.3-8.6 \mathrm{~s}$, mean 3.7 [SD: 4.0] s (supplementary table 1). In this group, maximal differences in site-1 annotated RF duration, FTI, ILD and impedance drop were $8.6 \mathrm{~s}, 208 \mathrm{~g} . \mathrm{s}, 7.7 \mathrm{~mm}$ and 1.4 respectively, with RMA "on" resulting in greater values for annotated data in $7 / 9$ transitions. When considering multiple measures of catheter position stability, the appropriate indication of deliberate catheter motion occurred with RMA "off" in all 9 transitions; 8/9 demonstrated [?]1s difference in annotation timing (supplementary figure 13).

For all 13 deliberate site 1-to-2 transitions achieved with constant catheter-tissue contact, the relationship between differences in annotated RF data (RMA "on" minus "off") and ITD (with RMA "off") is shown in figure 3. There was a strong negative correlation between the difference in annotated RF duration and ITD - Pearson $\mathrm{r}-0.68$ (95\% confidence interval (CI) -0.91 to - 0.13 , $\mathrm{p}=0.02$ ). Consequently, while the maximum difference in annotated RF duration with [?]6 $\mathrm{mm}$ site 1-to-2 ITD was 1.1s, [?]5 $\mathrm{mm}$ ITD was associated with maximal difference in annotated RF duration of 11.3s. There was a moderate negative correlation between the difference in annotated FTI and ITD - Pearson $\mathrm{r}-0.47$ (95\% CI -0.81 to $0.11, \mathrm{p}=0.10)$ and a moderate negative correlation between the difference in impedance drop and ITD - Pearson $\mathrm{r}-0.53$ (95\% CI -0.84 to $0.05, \mathrm{p}=0.07)$.

Data supplement figures 7 - 15 demonstrate remaining annotated site 1-to-2 transitions, with corresponding position shift, SD, CF and impedance data.

\section{Analyses at subsequently annotated LAPW sites during continuous RF delivery}

All left-sided LAPW lesion sets and 6 of 8 right-sided LAPW lesion sets were completed during continuous, uninterrupted RF application; onset timing of transitions for annotated sites 2-to-3, 3-to-4 and 4-to-5 is shown in supplementary table 2. At the left-sided LAPW there was progressively greater difference in timing between annotated sites of transition with RMA "on" versus "off", increasing from a median of $1.2 \mathrm{~s}$ (IQR $0.4-5.0$ ) at annotated site-2 onset, to a median of 14.6s (IQR 7.6 - 23.0) at annotated site-5 onset. Examples for one case are shown in supplementary figures 15-18. At the right-sided LAPW, there was no progressive in crease in difference between RMA "on" versus "off" annotation. However, 35\% (95\% CI 21-53) 
right-sided transitions occurred with intermittent catheter-tissue contact, whereas for left-sided sites this was only $3 \%$ (95\% CI $0-17)$.

\section{Discussion}

The main findings of these investigations into RMA use during automated RF annotation at the LAPW are:

At sites of deliberate catheter motion between first and second auto-annotated sites effected without loss of catheter-tissue contact, RMA "off" demonstrated suitable accuracy towards catheter motion detection in all cases.

Conversely, RMA "on" resulted in delayed identification of true catheter tip motion, resulting in delayed autoannotation of site 1-to-2 transition and important error in per-site RF parameters. Put simply, RMA "on" effectively renders automated RF annotation "blind" to the immediate occurrence of clinically important catheter displacement events.

These results may be better understood when considering the VISITAG Module annotation "system logic" and how this is modified by RMA use. Briefly, the ablation catheter tip position is measured in a "rolling window" of 60 sites per second (i.e. intervals of $16 / 17 \mathrm{~ms}$ ), from which is calculated the standard deviation (SD). With RMA (i.e. ACCURESP) "off", RF auto-annotation occurs when both every 1s of position data is within twice $(2 \mathrm{x})$ the user-defined position $\mathrm{SD}$, and a total consecutive minimum of $3 \mathrm{~s}$ is within $(1 \mathrm{x})$ the SD. However, with RMA "on", the position stability filter operates over a minimum of two respiratory cycles, using position data "adjusted" to end-expiration. In this situation, automated RF annotation occurs when these position stability targets are met following data adjustment. Importantly, annotation only occurs when CF filter preferences are also satisfied: in contrast to position stability filtering, the CF filter is applied continuously regardless of RMA setting (i.e. independent of the respiratory cycle). Therefore, with the force-over-time 100\% minimum $1 \mathrm{~g} \mathrm{CF}$ filter used during this present report, RF annotation "end" logic is fulfilled by any $\mathrm{Og}$ CF event at any stage in the respiratory cycle - i.e. regardless of RMA setting.

\section{Importance of these findings: Historical perspective}

Automated RF annotation has been shown to allow both the derivation of hypothetically ideal "per-site" target RF ablation parameters and their subsequent delivery during CF-guided PVI. ${ }^{3-5,8}$ However, such parameters can only be considered appropriate if "derivation phase" methodology employed a suitable definition for stable sites of catheter-tissue interaction during RF application. Notwithstanding the theoretical difficulty resulting from any CF filter permitting intermittent catheter-tissue contact (i.e. by definition a stable site can only occur in the setting of constant catheter-tissue contact), the foundational study supporting a regional difference in ablation target values additionally used ACCURESP RMA "on" in all cases; procedures performed under GA and with IPPV failing to trigger motion detection had the tidal volume increased to ensure respiratory motion triggering and RMA "on" use in all (Molloy Das, personal communication). ${ }^{5}$ Following this present report's demonstration that RMA use during automated RF annotation results in the failure to immediately identify catheter tip movements of up to $7 \mathrm{~mm}$, these previously identified ablation targets are likely to be importantly flawed.

\section{Clinical importance in light of novel high power, short per-site RF duration protocols}

RF lesion formation occurs more rapidly at higher power. ${ }^{11}$ Recently reported high power short duration (HPSD) protocols (50W, typically ${ }^{\sim} 5 \mathrm{~s}$ per site $)^{12,13}$ are attractive for operators wishing to move away from the requirement to maintain a stable catheter position for the ${ }^{\sim} 20-40$ s previously considered necessary to achieve transmural RF effect during PVI. ${ }^{1,14}$ However, at greater rates of lesion formation, methods to immediately and accurately identify clinically relevant catheter position instability are of particular importance towards knowing attainment of target "per-site" RF delivery and reproducible protocols. However, to our knowledge no HPSD RF manuscript includes details of RMA use. Alongside the recommendation for RF annotation use (i.e. VISITAG) with RMA "on", this also raises the question of the validity of HPSD "per-site" ablation targets, and whether presently described HPSD RF protocols may be considered reproducible. 


\section{Future directions for research}

For VISITAG RF annotation-guided procedures, another important determinant of auto-annotation logic towards a suitable definition for stable catheter-tissue interaction is the choice of position stability filter setting. Indeed, a possible solution to the inaccuracy of the RMA "on" setting as described here, is to use a smaller position stability filter range - e.g. $1.5 \mathrm{~mm}$. Although a complete description of the effects of this position filter setting is beyond the scope of this present report, data supplement figure 19 demonstrates left PV annotated site-1 data for case \#5 (reconstituted data curves in figure 2), using the RMA "off" with $2 \mathrm{~mm}$ position stability, and RMA "on" with $1.5 \mathrm{~mm}$ position stability range. Importantly, when using $1.5 \mathrm{~mm}$ range with RMA "on", annotated RF duration at site-1 is 1.6s greater, yet as shown in figure 2, position stability and CF changes indicating catheter displacement coincide with annotation using RMA "off" and $2 \mathrm{~mm}$ range (blue vertical line). In this case, further evidence towards this earlier time point representing the true site of catheter displacement is provided by the UE morphology change from pure R (site-1 end) to RS (site-2 onset) coinciding with RMA "off" and $2 \mathrm{~mm}$ range annotation timing. Clearly, such a delay to recognition of true catheter motion of $1.6 \mathrm{~s}$ is likely to be clinically important, particularly when considering HPSD ablation.

Together with a steerable sheath for catheter support, high frequency jet ventilation (HFJV) has been shown to reduce the occurrence of acute and chronic pulmonary vein reconnections and improve freedom from AF. ${ }^{15}$ Theoretically, complete absence of respiratory motion not only eliminates the requirement for respiratory adjustment, but also confers the advantage of eliminating any operator and/or patient-specific requirement for adjustments to sheath and/or catheter position according to the respiratory cycle. Accordingly, RF annotation-guided PVI protocols using HFJV may demonstrate greater reproducibility and efficacy.

\section{Limitations}

This report was of a single operator's practice, with analyses limited to annotated LAPW sites of RF delivery in view of the risk of atrio-oesophageal fistula resulting from excessive RF application at this site. It is possible that RMA settings demonstrate greater annotation concordance at alternative left atrial sites, however such analyses were beyond the scope of this present report. The VISITAG Module filter preferences (force-over-time $100 \%$ minimum $1 \mathrm{~g}$, with $2 \mathrm{~mm}$ position stability), overdrive atrial pacing and steerable sheath use towards achieving catheter stability and optimal ITD during continuous RF application must be taken into account when considering these experimental findings. If the more commonly employed force-over-time $30 \%$ minimum 4-5g CF filter was employed ${ }^{4,5}$, intermittent catheter-tissue contact would only trigger per-site annotation "end" if position data breached the chosen (position) stability criteria at that time-point - i.e. the effect of RMA "on" towards failure to identify sites of true catheter motion would be even greater. We elected not to perform a complete re-analysis of exported data with this different CF filter setting, since a site of stable catheter-tissue interaction during RF by definition may only occur in the setting of constant catheter-tissue contact (i.e. assuming no catheter CF measurement error, force-over-time $100 \%$ [?]1g). Also, a complete description of the effects of changing the position stability filter setting is beyond the scope of this present report; this will be the subject of a future manuscript.

Catheter tip motion characteristics following deliberate movement may differ from unintentional events: due to the very high attainment of target per-site RF delivery in this present report, a description of position stability, CF and impedance profiles at sites of accidental catheter motion is beyond the scope of this present report. However, when deliberate catheter displacement events of up to $\sim 5-7 \mathrm{~mm}$ were not immediately identified when annotated with RMA "on", similar degrees of movement during unintentional catheter displacement events are also likely to be missed.

The findings of this present report are only applicable to VISITAG auto-annotation guided PVI. However, EnSite Precision (Abbott) - the only other system presently employing automated RF annotation, AutoMark - also routinely utilises respiratory motion "compensation". Accordingly, the findings of this present report should stimulate investigations into the methodological rigour of "respiratory compensation" during AutoMark-guided AF ablation. 
Finally, this report is based on analyses of just 8 PVI procedures. However, RF annotation use provides a highly "data-rich" operative environment for analysis, and each 15s first-site annotated RF application alone provided 3090 exported data points (i.e. CF at $20 \mathrm{~Hz}$, impedance at $10 \mathrm{~Hz}$, and both position SD and position shift at $60 \mathrm{~Hz}$ ): this present report represents a total analysis of $\sim 165,000$ mapping system-derived data points.

\section{Conclusions}

ACCURESP RMA creates important error during automated RF annotation at the LAPW, via delayed identification of deliberate and clinically important catheter motion events. Previously identified autoannotated ablation targets using RMA "on" are likely to be importantly flawed, and on-going RMA use represents an important impediment towards greater reproducibility during PVI. In contrast, automated RF annotation with RMA "off" demonstrated excellent catheter motion detection. We therefore recommend avoiding RMA use during automated RF annotation guided PVI.

\section{Acknowledgements}

I am grateful to Cherith Wood, Daniel Newcomb and Ian Lines, Cardiac Physiologists, for their technical support into all cases conducted during this report. I am also grateful to Robert Pearce and Vicky Healey (Biosense Webster Inc.) for additional technical assistance and to Noam Seker-Gafni, Tal Bar-on, Einav Geffen, Assaf Rubissa and colleagues at the Haifa Technology Center, Israel (Biosense Webster Inc.) for their help with VISITAG Module technical queries.

\section{References}

1. Pappone C, Rosanio S, Oreto G, et al.: Circumferential radiofrequency ablation of pulmonary vein ostia: A new anatomic approach for curing atrial fibrillation. Circulation [Internet] 2000 [cited 2017 Nov 20]; 102:2619-2628. Available from: http://www.ncbi.nlm.nih.gov/pubmed/11085966

2. Ouyang F, Antz M, Ernst S, et al.: Recovered pulmonary vein conduction as a dominant factor for recurrent atrial tachyarrhythmias after complete circular isolation of the pulmonary veins: lessons from double Lasso technique. Circulation [Internet] 2005 [cited 2015 Nov 12]; 111:127-135. Available from: http://www.ncbi.nlm.nih.gov/pubmed/15623542

3. Hussein A, Das M, Chaturvedi V, et al.: Prospective use of Ablation Index targets improves clinical outcomes following ablation for atrial fibrillation. J Cardiovasc Electrophysiol [Internet] 2017 [cited 2017 Nov 20]; 28:1037-1047. Available from: http://www.ncbi.nlm.nih.gov/pubmed/28639728

4. Taghji P, El Haddad M, Phlips T, et al.: Evaluation of a Strategy Aiming to Enclose the Pulmonary Veins With Contiguous and Optimized Radiofrequency Lesions in Paroxysmal Atrial Fibrillation: A Pilot Study. JACC Clin Electrophysiol 2018; 4:99-108.

5. Das M, Loveday JJ, Wynn GJ, et al.: Ablation index, a novel marker of ablation lesion quality: prediction of pulmonary vein reconnection at repeat electrophysiology study and regional differences in target values. Europace [Internet] 2016 [cited 2016 Sep 7]; . Available from: http://www.ncbi.nlm.nih.gov/pubmed/27247002

6. Tomlinson D, Myles M, Stevens K, Streeter AJ: Transmural unipolar electrogram morphology is achieved within $7 \mathrm{~s}$ at the posterior left atrial wall during pulmonary vein isolation: VISITAG ${ }^{\mathrm{TM}}$ Module-based lesion assessment during radiofrequency ablation. bioRxiv [Internet] Cold Spring Harbor Laboratory, 2017 [cited 2017 Dec 28]; :234799. Available from: https://www.biorxiv.org/content/early/2017/12/15/234799

7. Calkins H, Hindricks G, Cappato R, et al.: 2017 HRS/EHRA/ECAS/APHRS/SOLAECE expert consensus statement on catheter and surgical ablation of atrial fibrillation. Hear Rhythm [Internet] 2017 [cited 2019 Jan 23]; 14:e275-e444. Available from: http://www.ncbi.nlm.nih.gov/pubmed/28506916

8. Tomlinson DR: Derivation and validation of a VISITAG ${ }^{\text {TM }}$-guided contact force ablation protocol for pulmonary vein isolation. bioRxiv [Internet] Cold Spring Harbor Laboratory, 2017 [cited 2017 Dec 14]; 
:232694. Available from: https://www.biorxiv.org/content/early/2017/12/13/232694

9. Tomlinson DR, Stevens KN, Streeter AJ: Assessment of the transmural unipolar electrogram morphology change radius during contact force-guided pulmonary vein isolation using the VISITAG $^{\text {TM }}$ Module and CARTOREPLAY ${ }^{\mathrm{TM}}$. bioRxiv [Internet] Cold Spring Harbor Laboratory, 2018 [cited 2018 Apr 25]; :284539. Available from: https://www.biorxiv.org/content/early/2018/03/19/284539.figures-only

10. R: A language and environment for statistical computing. R Core Team, Foundation for Statistical Computing, Vienna, Austria. 2017; :https://www.r-project.org/.

11. Irastorza RM, d'Avila A, Berjano E: Thermal latency adds to lesion depth after application of high-power short-duration radiofrequency energy: Results of a computer-modeling study. J Cardiovasc Electrophysiol [Internet] 2018 [cited 2019 Jan 23]; 29:322-327. Available from: http://www.ncbi.nlm.nih.gov/pubmed/28988468

12. Chelu MG, Morris AK, Kholmovski EG, et al.: Durable lesion formation while avoiding esophageal injury during ablation of atrial fibrillation: Lessons learned from late gadolinium MR imaging. J Cardiovasc Electrophysiol [Internet] 2018 [cited 2019 Jan 23]; 29:385-392. Available from: http://www.ncbi.nlm.nih.gov/pubmed/29345381

13. Winkle RA, Moskovitz R, Hardwin Mead R, et al.: Atrial fibrillation ablation using very short duration $50 \mathrm{~W}$ ablations and contact force sensing catheters. J Interv Card Electrophysiol [Internet] 2018 [cited 2019 Jan 23]; 52:1-8. Available from: http://www.ncbi.nlm.nih.gov/pubmed/29460232

14. Arentz T, Weber R, Burkle G, et al.: Small or Large Isolation Areas Around the Pulmonary Veins for the Treatment of Atrial Fibrillation?: Results From a Prospective Randomized Study. Circulation [Internet] 2007 [cited 2017 Nov 20]; 115:3057-3063. Available from: http://www.ncbi.nlm.nih.gov/pubmed/17562956

15. Hutchinson MD, Garcia FC, Mandel JE, et al.: Efforts to enhance catheter stability improve atrial fibrillation ablation outcome. Heart Rhythm [Internet] 2013 [cited 2015 Nov 12]; 10:347-353. Available from: http://www.ncbi.nlm.nih.gov/pubmed/23128019

\section{Tables}

Table 1: Biophysical data at auto-annotated sites of RF delivery at the LAPW according to ACCURESP setting (i.e. RMA "on" versus "off") and site; data shown are mean $[\mathrm{SD}]$ or median $\left(1^{\text {st }}-3^{\text {rd }}\right.$ quartile), as appropriate. Note: "Number of annotated sites" data represents the mean of 8 patients; ${ }^{+}$indicates the number of LAPW annotated sites identified and used for analyses of the remaining variables (ITD, inter-tag distance; FTI, force time integral).

Table 2: Auto-annotated data at first-annotated LAPW sites according to ACCURESP setting (i.e. RMA "on" versus "off") and site; data shown are median ( $1^{\text {st }}-3^{\text {rd }}$ quartile).

Table 3: Auto-annotated data at site 1-to-2 transition, with deliberate catheter movement identified according to criteria of a $0 \mathrm{~g} C F$ event, or change in the UE morphology from pure R (site- 1 completion) to RS (site-2 onset). Auto-annotated site-1 RF duration, site 1-to-2 ILD, impedance drop and FTI data are displayed according to RMA setting, with the difference ("Diff" - i.e. RMA "on" minus "off") also shown; PV, pulmonary vein.

\begin{tabular}{|c|c|c|c|c|c|c|c|}
\hline $\begin{array}{l}\text { Left- } \\
\text { sided } \\
\text { anno- } \\
\text { tated } \\
\text { sites }\end{array}$ & $\begin{array}{l}\text { Left- } \\
\text { sided } \\
\text { anno- } \\
\text { tated } \\
\text { sites }\end{array}$ & $\begin{array}{l}\text { Left- } \\
\text { sided } \\
\text { anno- } \\
\text { tated } \\
\text { sites }\end{array}$ & $\begin{array}{l}\text { Right- } \\
\text { sided } \\
\text { anno- } \\
\text { tated } \\
\text { sites }\end{array}$ & $\begin{array}{l}\text { Right- } \\
\text { sided } \\
\text { anno- } \\
\text { tated } \\
\text { sites }\end{array}$ & $\begin{array}{l}\text { Right- } \\
\text { sided } \\
\text { anno- } \\
\text { tated } \\
\text { sites }\end{array}$ & $\begin{array}{l}\text { All } \\
\text { anno- } \\
\text { tated } \\
\text { sites }\end{array}$ & $\begin{array}{l}\text { All } \\
\text { anno- } \\
\text { tated } \\
\text { sites }\end{array}$ \\
\hline $\begin{array}{l}\text { RMA } \\
\mathrm{ON} \\
\left(\mathrm{N}=43^{+}\right)\end{array}$ & $\begin{array}{l}\text { RMA } \\
\mathrm{OFF} \\
\left(\mathrm{N}=52^{+}\right)\end{array}$ & $p$ & $\begin{array}{l}\text { RMA } \\
\text { ON } \\
\left(\mathrm{N}=39^{+}\right)\end{array}$ & $\begin{array}{l}\text { RMA } \\
\text { OFF } \\
\left(\mathrm{N}=46^{+}\right)\end{array}$ & $p$ & $\begin{array}{l}\text { RMA } \\
\mathrm{ON} \\
\left(\mathrm{N}=82^{+}\right)\end{array}$ & $\begin{array}{l}\mathrm{RMA} \\
\mathrm{OFF} \\
\left(\mathrm{N}=98^{+}\right)\end{array}$ \\
\hline
\end{tabular}




\begin{tabular}{|c|c|c|c|c|c|c|c|c|c|}
\hline & $\begin{array}{l}\text { Left- } \\
\text { sided } \\
\text { anno- } \\
\text { tated } \\
\text { sites }\end{array}$ & $\begin{array}{l}\text { Left- } \\
\text { sided } \\
\text { anno- } \\
\text { tated } \\
\text { sites }\end{array}$ & $\begin{array}{l}\text { Left- } \\
\text { sided } \\
\text { anno- } \\
\text { tated } \\
\text { sites }\end{array}$ & $\begin{array}{l}\text { Right- } \\
\text { sided } \\
\text { anno- } \\
\text { tated } \\
\text { sites }\end{array}$ & $\begin{array}{l}\text { Right- } \\
\text { sided } \\
\text { anno- } \\
\text { tated } \\
\text { sites }\end{array}$ & $\begin{array}{l}\text { Right- } \\
\text { sided } \\
\text { anno- } \\
\text { tated } \\
\text { sites }\end{array}$ & $\begin{array}{l}\text { All } \\
\text { anno- } \\
\text { tated } \\
\text { sites }\end{array}$ & $\begin{array}{l}\text { All } \\
\text { anno- } \\
\text { tated } \\
\text { sites }\end{array}$ & \\
\hline $\begin{array}{l}\text { Number of } \\
\text { sites per } \\
\text { patient }\end{array}$ & $5.4[1.3]$ & $6.5[1.6]$ & 0.15 & $4.9[1.5]$ & $5.8[1.7]$ & 0.28 & $5.1[1.4]$ & $6.1[1.6]$ & \\
\hline ITD (mm) & $5.6[1.6]$ & $4.6[1.7]$ & 0.01 & $6.2[2.2]$ & $5.4[2.6]$ & 0.16 & $\begin{array}{l}6.0(4.7- \\
6.8)\end{array}$ & $\begin{array}{l}4.8(3.9- \\
5.6)\end{array}$ & \\
\hline $\begin{array}{l}\mathrm{RF} \\
\text { duration } \\
\text { (s) }\end{array}$ & $13.1[4.4]$ & $9.9[3.9]$ & 0.0003 & $13.5[5.5]$ & $10.6[3.9]$ & 0.006 & $\begin{array}{l}13.3(10.3 \\
-15.6)\end{array}$ & $\begin{array}{l}10.6(7.5- \\
13.2)\end{array}$ & \\
\hline $\begin{array}{l}\text { Mean CF } \\
(\mathrm{g})\end{array}$ & $\begin{array}{l}12.1(9.9- \\
15.6)\end{array}$ & $\begin{array}{l}11.5(9.7- \\
14.9)\end{array}$ & 0.67 & $\begin{array}{l}17.9(13.4 \\
-21.6)\end{array}$ & $\begin{array}{l}18.6(12.1 \\
-22.1)\end{array}$ & 0.68 & $\begin{array}{l}14.4(10.8 \\
-19.7)\end{array}$ & $\begin{array}{l}14.0(10.7 \\
-19.9)\end{array}$ & \\
\hline $\begin{array}{l}\text { Impedance } \\
\text { drop () }\end{array}$ & $\begin{array}{l}8.0(4.3- \\
12.2)\end{array}$ & $\begin{array}{l}6.6(3.4- \\
10.6)\end{array}$ & 0.24 & $\begin{array}{l}5.9(1.5- \\
8.8)\end{array}$ & $\begin{array}{l}3.7(0.8- \\
7.1)\end{array}$ & 0.34 & $\begin{array}{l}6.5(3.5- \\
10.0)\end{array}$ & $\begin{array}{l}5.4(1.7- \\
8.9)\end{array}$ & \\
\hline FTI (g.s) & $\begin{array}{l}156(123- \\
204)\end{array}$ & $\begin{array}{l}114(31- \\
161)\end{array}$ & 0.0003 & $\begin{array}{l}228(134- \\
315)\end{array}$ & $\begin{array}{l}166(111- \\
243)\end{array}$ & 0.04 & $\begin{array}{l}177(133- \\
250)\end{array}$ & $\begin{array}{l}130(104- \\
195)\end{array}$ & \\
\hline
\end{tabular}

Table 1

\begin{tabular}{|c|c|c|c|c|c|c|c|c|c|}
\hline & $\begin{array}{l}\text { Left- } \\
\text { sided } \\
\text { site-1 }\end{array}$ & $\begin{array}{l}\text { Left- } \\
\text { sided } \\
\text { site-1 }\end{array}$ & $\begin{array}{l}\text { Left- } \\
\text { sided } \\
\text { site-1 }\end{array}$ & $\begin{array}{l}\text { Right- } \\
\text { sided } \\
\text { site-1 }\end{array}$ & $\begin{array}{l}\text { Right- } \\
\text { sided } \\
\text { site-1 }\end{array}$ & $\begin{array}{l}\text { Right- } \\
\text { sided } \\
\text { site-1 }\end{array}$ & $\begin{array}{l}\text { Site-1: } \\
\text { All } \\
\text { data }\end{array}$ & $\begin{array}{l}\text { Site-1: } \\
\text { All } \\
\text { data }\end{array}$ & \\
\hline & RMA & RMA & \multirow[t]{2}{*}{$p$} & RMA & \multirow{2}{*}{$\begin{array}{l}\text { RMAOFF } \\
(\mathrm{N}=8)\end{array}$} & \multirow[t]{2}{*}{$p$} & RMA & RMA & \\
\hline & $\begin{array}{l}\mathrm{ON} \\
(\mathrm{N}=8)\end{array}$ & $\begin{array}{l}\mathrm{OFF} \\
(\mathrm{N}=8)\end{array}$ & & $\begin{array}{l}\mathrm{ON} \\
(\mathrm{N}=8)\end{array}$ & & & $\begin{array}{l}\mathrm{ON} \\
(\mathrm{N}=16)\end{array}$ & $\begin{array}{l}\mathrm{OFF} \\
(\mathrm{N}=16)\end{array}$ & \\
\hline $\begin{array}{l}\text { Site } 1 \text {-to-2 } \\
\text { ITD (mm) }\end{array}$ & $\begin{array}{l}6.6(6.3- \\
7.0)\end{array}$ & $\begin{array}{l}5.3(4.6- \\
6.3)\end{array}$ & 0.07 & $\begin{array}{l}7.2(6.3- \\
8.4)\end{array}$ & $\begin{array}{l}5.0(4.5- \\
8.1)\end{array}$ & 0.13 & $\begin{array}{l}6.7(6.3- \\
7.8)\end{array}$ & $\begin{array}{l}5.2(4.5- \\
6.8)\end{array}$ & \\
\hline $\mathrm{RF}$ & $16.0(15.2$ & $15.1(14.7$ & 0.16 & $15.5(14.7$ & $15.1(14.7$ & 0.57 & $15.7(14.9$ & 15.1 & \\
\hline $\begin{array}{l}\text { duration } \\
\text { (s) }\end{array}$ & $-18.7)$ & $-15.6)$ & & $-23.7)$ & $-15.7)$ & & $-22.2)$ & $-15.6)$ & \\
\hline $\begin{array}{l}\text { Mean CF } \\
(\mathrm{g})\end{array}$ & $\begin{array}{l}10.8(9.5- \\
12.9)\end{array}$ & $\begin{array}{l}10.8(9.0- \\
13.0)\end{array}$ & 0.88 & $\begin{array}{l}16.8(12.6 \\
-21.6)\end{array}$ & $\begin{array}{l}17.8(11.7 \\
-21.2)\end{array}$ & 1.00 & $\begin{array}{l}12.9(10.1 \\
-18.2)\end{array}$ & $\begin{array}{l}13.0(9.0- \\
18.3)\end{array}$ & \\
\hline Impedance & $\begin{array}{l}13.5(11.5 \\
-23.6)\end{array}$ & $\begin{array}{l}13.5(10.9 \\
-21.5)\end{array}$ & 0.80 & $\begin{array}{l}10.3(8.5- \\
12.4)\end{array}$ & $\begin{array}{l}10.3(7.8- \\
12.4)\end{array}$ & 0.96 & $\begin{array}{l}12.0(9.1- \\
15.7)\end{array}$ & $\begin{array}{l}11.5(9.1- \\
15.2)\end{array}$ & \\
\hline FTI (gs) & $\begin{array}{l}185(150 \text { - } \\
230)\end{array}$ & $\begin{array}{l}163(123- \\
198)\end{array}$ & 0.33 & $\begin{array}{l}268(240- \\
376)\end{array}$ & $\begin{array}{l}264(185- \\
327)\end{array}$ & 0.65 & $\begin{array}{l}240(163- \\
322)\end{array}$ & $\begin{array}{l}198(135- \\
270)\end{array}$ & \\
\hline
\end{tabular}

Table 2

\begin{tabular}{llllll}
\hline Case number & PV & Position instability criterion & Site-1 RF duration (s) & Site-1 RF duration (s) & Site- \\
\hline & & & RMA & RMA & RMA \\
& & & ON & OFF & Diff \\
5 & Right & Og CF & 15.5 & 15.6 & -0.1 \\
10 & Right & Og CF & 14.4 & 14.4 & 0 \\
11 & Right & Og CF & 14.0 & 14.6 & -0.6
\end{tabular}




\begin{tabular}{llllll}
\hline Case number & PV & Position instability criterion & Site-1 RF duration (s) & Site-1 RF duration (s) & Site- \\
\hline 5 & Left & Pure R UE to RS & 26.5 & 15.2 & 11.3 \\
11 & Left & Pure R UE to RS & 20.9 & 18.6 & 2.3 \\
22 & Right & Pure R UE to RS & 15.1 & 14.8 & 0.3 \\
23 & Right & Pure R UE to RS & 15.5 & 15.0 & 0.5 \\
\hline
\end{tabular}

Table 3

Figures

Figure 1: Reconstituted curves from VISITAG Module data export demonstrating first-annotated site "end" time-point (i.e. site 1-to-2 transition) at the first $0 \mathrm{~g}$ CF event, 15.5s (ACCURESP "on") and 15.6s (ACCURESP "off") following RF onset in case 5, right PV. This small difference in the annotated RF duration between ACCURESP settings results in a single vertical red line representing ACURESP "on" and "off" annotation timing. Catheter tip position shift, (position) standard deviation (SD), CF and impedance are plotted separately; x-axis displays the PIU time stamp (i.e. running case time, shown in thousands of seconds) and vertical dashed lines indicate end-expiration time-points. The force-over-time $100 \%$ minimum $1 \mathrm{~g}$ CF filter ensures that automated RF annotation only occurs when CF is continuously maintained [?]1g, indicated by black curves; pink position shift, SD and impedance curves indicate $<100 \%$ CF filter preference attainment. The SD curve returns to black at 1s (or 60 sites) following attainment of both CF and position stability within the chosen VISITAG Module filter preferences, in view of the CARTO ${ }^{\circledR} 3$ SD calculation "system logic". Note: "Session 200 at sites 41 and 42" represents the unique automatically generated VISITAG Module annotated identifiers for this site.

Figure 2: Reconstituted curves from VISITAG Module data export demonstrating first-annotated site "end" time-point (i.e. site 1-to-2 transition) at 15.2s (ACCURESP "off", blue line) and 26.5s (ACCURESP "on", red line) following RF onset in case 5, left PV. Transition to site-2 with ACCURESP "off" coincided with UE morphology change from pure R at site-1 completion, to RS at site-2 onset (ACCURESP "off" ITD 4.1mm); clear changes in the catheter tip position shift, (position) standard deviation (SD) and CF coincide with ACCURESP "off" annotation. All curves are drawn black, since CF was maintained [?]1g and the catheter movement was sufficiently rapid to ensure that all catheter tip location data was annotated to either site-1, or 2 (ACCURESP "off"). Note: "Session 181 at sites 1 and 2" represents the unique automatically generated VISITAG Module annotated identifiers for this site.

Figure 3: ACCURESP RMA "on" minus "off" difference in annotated RF duration, FTI and impedance drop according to site 1-to-2 ITD with ACCURESP "off". Data are shown for all 13 LAPW inter-ablation site transitions achieved during constant catheter-tissue contact, with corresponding correlation data. 

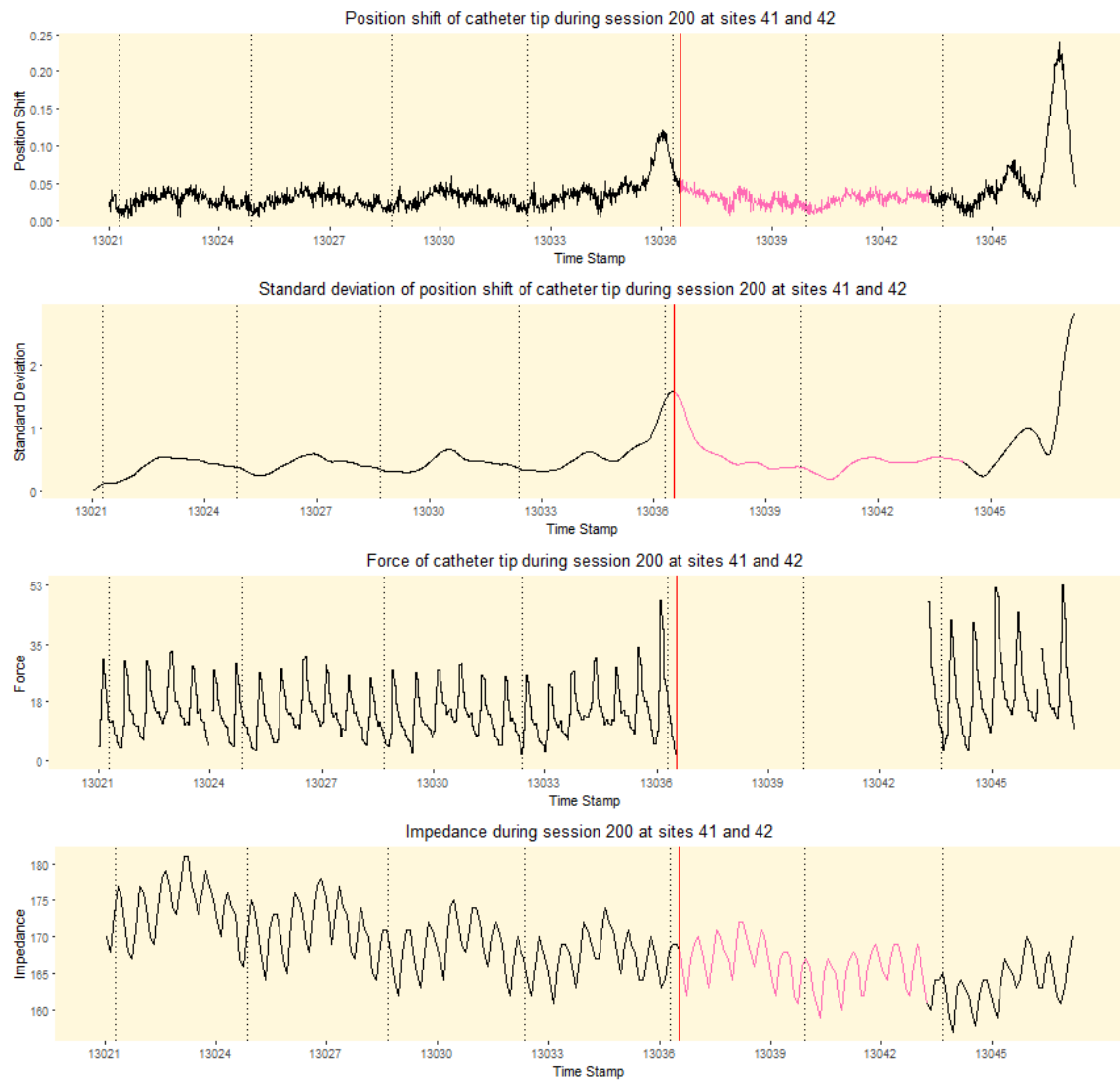

Figure 1 

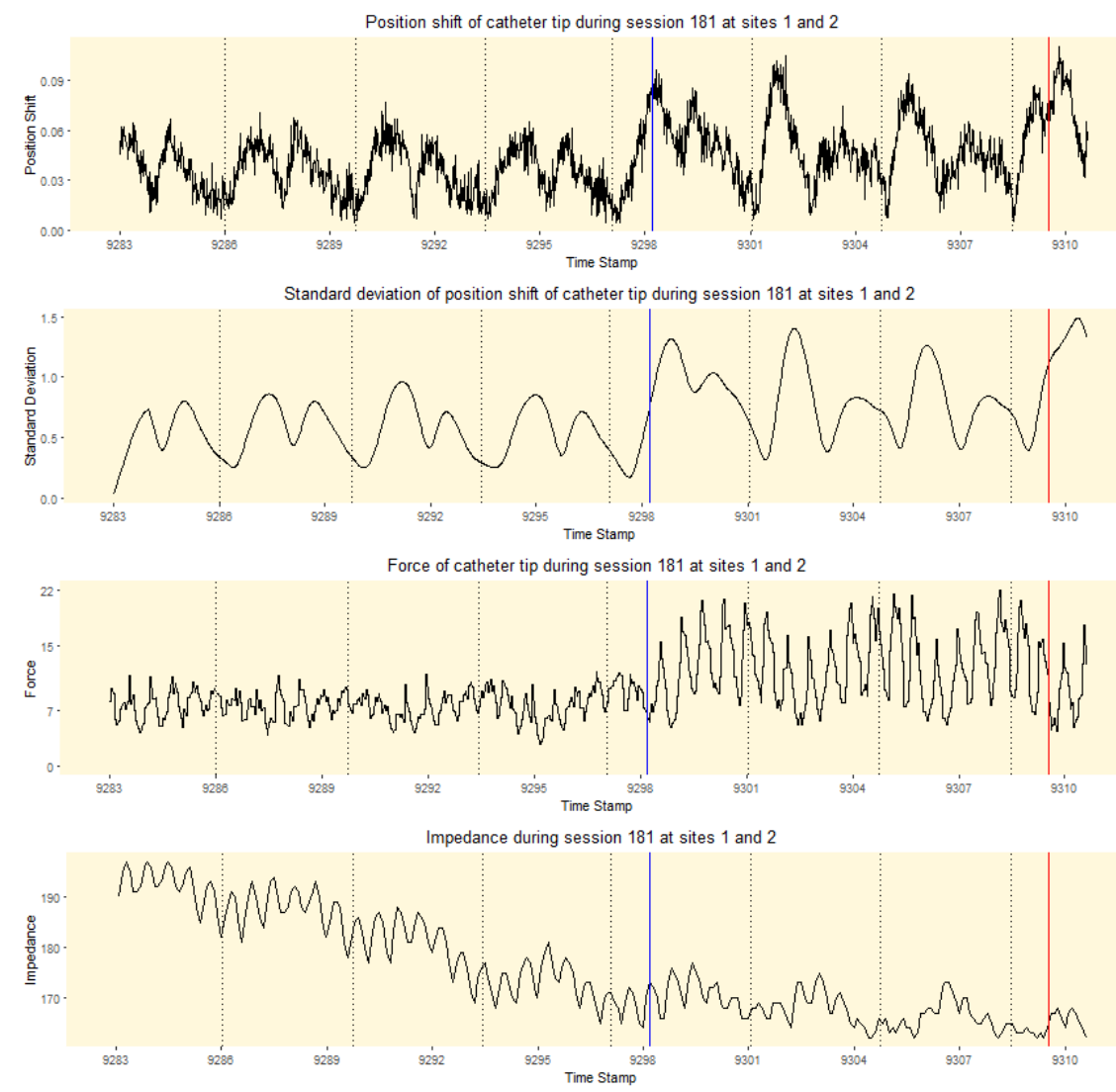

Figure 2

\section{Hosted file}

image3.emf available at https://authorea.com/users/379074/articles/495426-identificationof-deliberate-catheter-motion-at-the-left-atrial-posterior-wall-during-pulmonary-veinisolation-validity-of-respiratory-motion-adjustment

Figure 3 